\title{
A 15 year old girl presented with rectal bleeding, growth failure, digital clubbing and mental retardation
}

\author{
Afsana Yasmin, Md. Rukunuzzaman, A. S. M. Bazlul Karim, Rafia Rashid, Md. Benzamin and \\ Bishnu Pada Dey
}

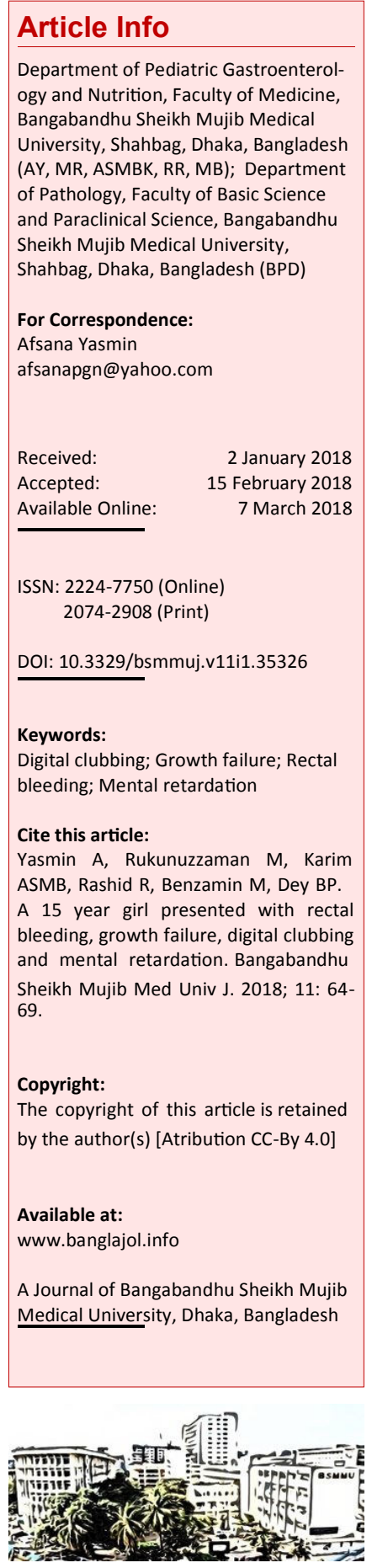

\section{Case Presentation}

Dr. Rafia Rashid: A 15 year old girl of non-comsanguineous parents presented at the outpatient department with the history of intermittent rectal bleeding for the last 6 years. Rectal bleeding was painless and there was the passage of a small amount of bright red blood drop by drop after defecation. She also complained of something coming out through the anus during defecation for last one year. Mother also noticed that her child had learning difficulties and not growing well like other peers. Her menarche had not started yet. Her elder two sisters and younger two brothers are healthy. She had no family history of rectal bleeding or colorectal cancer. She had no history of fever, abdominal pain, alteration of bowel habit, oral ulceration, weight loss and any joint manifestations. On examination, she was afebrile, mildly pale and vitally stable. Digital clubbing was present and skin finding was normal (Figure 1). Her height was $129 \mathrm{~cm}$ (severely stunted), mid-parental height $158 \mathrm{~cm}$, target height $148-168 \mathrm{~cm}$ and weight $25 \mathrm{~kg}$ (underweight). On rectal examination, there were a few polypoid masses in the rectum. Examination of the cardiovascular, neurological and other systems revealed no abnormality. Complete blood count with erythrocyte sedimentation rate, C-reactive protein, serum albumin, prothrombin time and activated partial thromboplastin time reports all were normal except mild anemia (Table I). Stool routine examination showed no feature of colitis.

After evaluating the clinical data, physical findings and investigations results, the case was provisionally diagnosed.

\section{Provisional Diagnosis}

Juvenile colorectal polyp

\section{Differential Diagnosis}

Dr. Md. Benzamin: Patient presented with fresh bright red rectal bleeding for long duration and something coming out through anal verge after defecation. Patients also had severe growth failure and learning difficulties. Though history was quite long but the patient did not need blood transfusion. The child was mildly pale. Digital clubbing and severe growth retardation were present. The important clue to the diagnosis of juvenile colorectal polyp is characteristic rectal bleeding. After evaluating the other features, I thought about another differential diagnosis of Inflammatory bowel disease.

\section{Inflammatory bowel disease}

Inflammatory bowel disease is a chronic inflammatory disease of intestine resulting from host-microbial interactions in a genetically susceptible individual. It is a group of autoimmune diseases where inflammation of any part of the small and large intestine may occur by the body's own immune system. It has two major forms, known as Crohn's disease and ulcerative colitis. 1 The clinical features of inflammatory bowel disease are abdominal pain, loose stools, weight loss, clubbing, aphthous ulcer and mucus with blood in stools. Rectal urgency and tenesmus during defecation may be present if the rectum is involved.2 Though the presence of rectal bleeding for prolonged period, growth failure and digital clubbing are suggestive of inflammatory bowel disease but the absence of diarrhea, abdominal pain and oral ulceration goes against the inflammatory bowel disease.

Dr. Afsana Yasmin: After evaluating the patient's presenting features, physical findings and the laboratory tests results, colonoscopy was done. Colonoscopy showed multiple polyps, about 30 mostly in the rectum and sigmoid colon and a few were in the descending and transverse colon. Most of the polyps were sessile and a few were pedunculated (Figure 1).

Dr. Bishnu Pada Dey: Histopathology of the polyp tissues showed hamartomatous polyp with no dysplasia or malignant cells (Figure 2).

Dr. Yasmin: We also did esophagogastroduodenoscopy which showed multiple sessile polyps in the stomach, mostly in the antrum with the escape of esophagus and duodenum (Figure 1). We also did ultrasonography of pelvic organs which showed infantile uterus. 


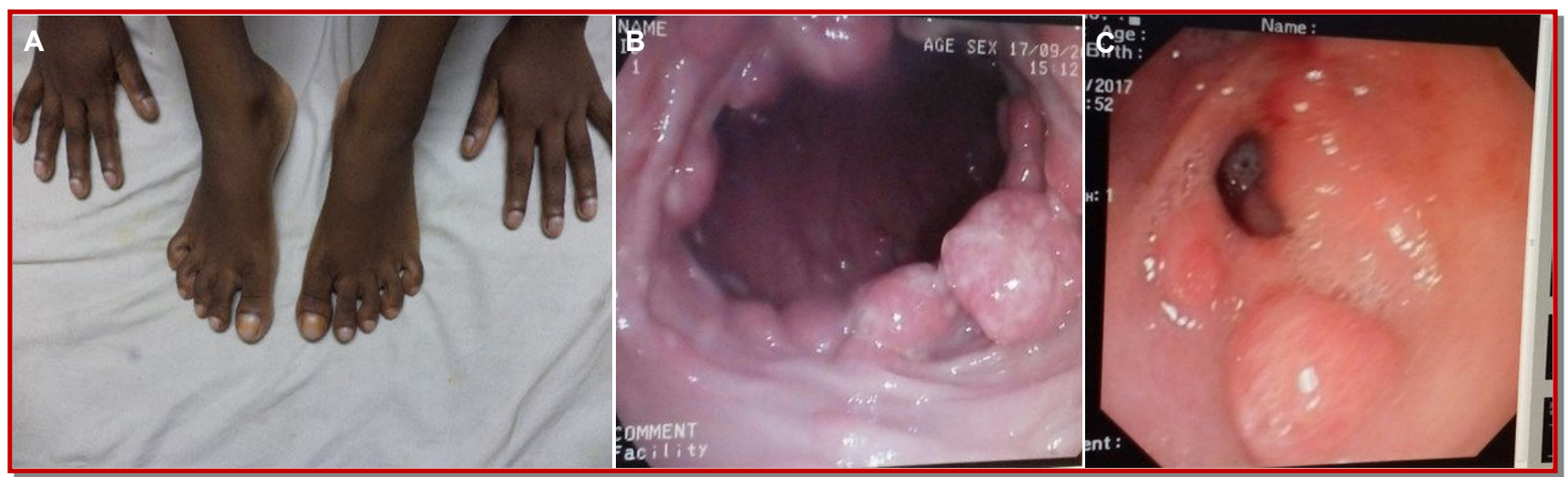

Figure 1: (A) Digital clubbing of the patient; (B) Colonoscopy shows multiple polyps in the colon and rectum; (C) Esophagogastroduodenoscopy shows multiple gastric polyps (C)

\begin{tabular}{|lrr|}
\hline \multicolumn{3}{|c|}{ Table I } \\
\hline \multicolumn{3}{|c|}{ Laboratory investigations } \\
\hline Investigation & Reference range & Patient's value \\
\hline Hemoglobin (g/dL) & $13.5 \pm 1.3$ & 11.6 \\
White cell count (x10\%/L) & $7.0 \pm 3.0$ & 9.8 \\
$\quad$ Neutrophil (\%) & $40-80$ & 54 \\
Lymphocyte (\%) & $20-40$ & 35 \\
Eosinophil (\%) & $1-6$ & 8 \\
$\quad$ Monocyte (\%) & $2-10$ & 3 \\
Platelet count (x10 $/ \mathrm{L})$ & $150-400$ & 20 \\
Erythrocyte sedimentation rate & $0-10$ & $<6$ \\
(mm in 1st hour) & & 36 \\
C-Reactive protein (mg/dL) & $<6$ & 30 \\
Serum albumin (g/dL) & $35-45$ & \\
Prothrombin time (sec) & 12 & \\
Activated partial thromboplastin & 28 & No growth of bacteria \\
time (sec) & & \\
Stool & & \\
$\quad$ Routine examination & & \\
Culture & & \\
\hline
\end{tabular}

Her luteinizing hormone was low and the follicle stimulating hormone was within normal range. Psychological assessment was done by the WISC-R which revealed mild mental retardation. According to the history, physical examination, colonoscopy findings and histopathology of polyp, it was the case of gastrointestinal hamartomatous polyposis syndrome. There are four types of hamartomatous polyposis syndrome including juvenile polyposis syndrome, Bannayan Riley Ruvalcaba syndrome, Cowden syndrome and Peutz-Jegher syndrome. The clinical manifestations of all polyposis syndromes share same characteristics of intestinal juvenile polyps.

\section{Juvenile polyposis syndrome}

Juvenile polyposis syndrome is a rare hamartomatous polyposis disorder with significant gastrointestinal malignant potential due to mutations in BMPR1A and SMAD4. 3 Juvenile polyposis syndrome patient presented with rectal bleeding, prolapsed rectal polyp, pallor, abdominal pain due to intussusception and diarrhea. Associated other morphological abnormalities are growth failure, alopecia, cleft lip or palate, macrocephaly, hypertelorism, polydactyly, digital clubbing, hydrocepha-

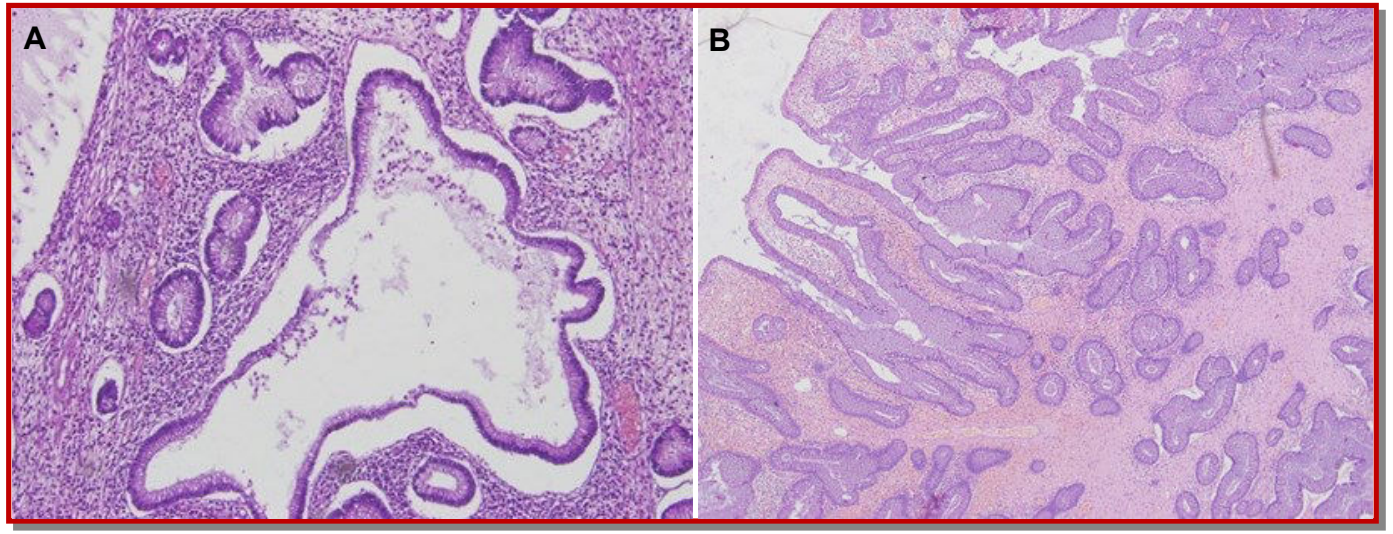

Figure 2: Hematoxylin and eosin staining of the (A) cystically dilated gland lined by intestinal mucosa (x200); (B) tubular adenoma with mild dysplasia 
lus, mental retardation, congenital cardiac anomalies, pulmonary arteriovenous malformations, pulmonary stenosis, telangiectasias, Meckel's diverticulum, intestinal malrotation, bifid uterus and vagina (female) and cryptorchidism (male). $\underline{4}$

If extraintestinal features consistent with Cowden syndrome or Bannayan Riley Ruvalcaba syndrome are absent, then the diagnosis of juvenile polyposis syndrome is made when the following clinical criteria are present: a) more than five juvenile polyps of the colon or rectum, or; b) juvenile polyps in other parts of the gastrointestinal tract, or; c) any number of juvenile polyps and a positive family history..$\underline{5}$

In the presenting case, presentations are rectal bleeding with associated abnormalities: growth failure, digital clubbing, mental retardation and infantile uterus, the presence of more than five juvenile polyps in the colorectum and also in the stomach. So, according to the JASS criteria, it was a case of juvenile polyposis syndrome if extraintestinal features of Cowden syndrome and Bannayan Riley Ruvalcaba syndrome were excluded.

\section{Cowden syndrome}

Cowden syndrome often is a rare hamartomatous polyposis syndrome caused by germline mutation in the tumor-suppressor gene phosphatase and tensin homolog.. Cowden syndrome is diagnosed on the basis of International operational diagnostic criteria. Pathognomic criteria are a) fascial trichilemmomas, b) acral keratosis, c) papillomatous papules and d) mucosal lesions. Major criteria are: a) breast carcinoma, b) thyroid carcinoma, c) macrocephaly and d) endometrial carcinoma. Minor criteria are: a) another thyroid lesion e.g. thyroid adenoma or multinodular goiter, b) mental retardation, c) gastrointestinal hamartoma, d) fibrocystic disease of the breast, e) lipoma, fibroma and f) genitorurinary tumor or malformation.

Operational diagnosis in a person: 1) Mucocutaneous lesions alone if: a) there are six or more facial papules, of which three or more must be trichilemmoma, or b) cutaneous facial papules and oral mucosal papillomatosis, or c) oral mucosal papillamatosis and acral keratoses, or d) palmoplantar keratoses, six or more; 2) two major criteria but one must include macrocephaly; 3 ) one major and three minor criteria; and 4) four minor criteria. 7

In our patients, there were no pathognomonic and major criteria. Only three minor criteria were there. The patient also had severe growth failure and digital clubbing which were features of juvenile polyposis syndrome.

\section{Bannayan Riley Ruvalcaba syndrome}

Bannayan Riley Ruvalcaba syndrome is another etiology of hamartomatous polyposis syndrome which is congenital and therefore unlike Cowden syndrome, clinical manifestations appear early in life. Common findings of Bannayan Riley Ruvalcaba syndrome include macrocephaly, high birth weight, proximal muscle myopathy $(60 \%)$, lipomatosis, intestinal polyposis, pigmented macules of the penis, joint hyperextensibility, pectus excavatum, scoliosis (50\%), as well as developmental delay and intellectual deficiency (50\%). $.8,9$ In this case presentation, no such type of findings except intellectual deficiency, rather there was the presence of growth failure and clubbing which are more in favor of juvenile polyposis syndrome.

\section{Dr. Yasmin's Diagnosis}

Juvenile polyposis syndrome

\section{Discussion}

Dr. Rukunuzzaman: Juvenile polyposis syndrome is a genetically predisposed condition. In $1964 \mathrm{McColl}$ first described juvenile polyposis syndrome.10 It may be caused by mutations in two genes: SMAD4/ DPC4 located on chromosome 18q21 and BMPR1A on chromosome 10q21-22.11, 12 Juvenile polyposis syndrome is an autosomal dominant condition like other hamartomatous polyposis and it follows a familial pattern from $20 \%$ to $50 \%$ of cases. Rest is sporadic. $\underline{13}$ The prevalence of juvenile polyposis syndrome is estimated 1:100000. .5 Alam et al (2017) showed in a study of 326 Bangladeshi children presented with per rectal bleeding, the incidence of juvenile polyposis syndrome is $1.5 \%$. 14 . Poddar et al (1998) showed on 236 Indian children with colonic polyps, $17(7 \%)$ were diagnosed as juvenile polyposis. $\underline{15}$

According to clinical presentation and disease course, juvenile polyposis syndrome patients further categorized into three phenotypic groups: a) juvenile polyposis of infancy; b) juvenile polyposis coli (colonic involvement only); and c) generalized juvenile polyposis (involve both small and large intestine)..$\underline{16}$ In juvenile polyposis syndrome, in order of frequency, the affected sites are colorectum $(98 \%)$, stomach $(14 \%)$, jejunum plus ileum $(7 \%)$ and duodenum $(2 \%) \cdot \underline{17}$

Gastrointestinal bleeding is the most common presentation of juvenile polyposis syndrome.18 Patients with juvenile polyposis coli present at ages between 5-15 years, whereas patients with generalized juvenile polyposis present at a younger age. 19 Associated other morphological abnormalities are found in $15 \%$ of cases, usually in those with no family history and include- growth failure, digital clubbing, polydactyly, alopecia, cleft lip or palate, cardiac defect, genitourinary defect like septate 
uterus or vagina and mental retardation, ataxia telangiectasia, malrotation of the midgut, and cranial defect. $\underline{5}$ The presenting case is a generalized juvenile polyposis syndrome in the sporadic form. She also had severe growth failure, clubbing, mental retardation and infantile uterus with the absence of secondary sexual characteristics along with per rectal bleeding. As far I know, it was the first case report where the patient did not develop secondary sexual characteristics with the infantile uterus in juvenile polyposis syndrome.

Most commonly affected site of polyps in juvenile polyposis syndrome is the colon, often have 50-200 polyps throughout the colon. The stomach and small intestine may also have polyps in a few patients. The size of polyps is variable ranges from a few millimeters to a few centimeters. $\frac{3}{-}$ The presenting case had about 40 polyps in colon and stomach.

Dr. Dey: Macroscopically, a juvenile polyp is smooth surfaced, red-colored sessile or pedunculated polypoid masses. Microscopically, numerous cystic and dilated crypts or glands with inspissated mucin and proliferative smaller glands located in the lamina propria. The glands are lined with normal intestinal mucosa. The Lamina propria is edematous and infiltrated with lymphocytes, plasma cells and occasional eosinophils and neutrophils. Muscularis mucosa is not included in the stroma. 5 Histological features of all hamartomatous polyposis syndromes share same characteristics except Peutz-Jegher syndrome where an extra feature is hyperplasia of smooth muscle layer which extends in tree-like manner toward the epithelial layer.20 Histological features were suggestive of hamartomatous polyp without evidence of dysplasia.

The colonic polyps had a similar pattern and were composed of elongated, sometimes branching glands. Some of them were cystically dilated and filled with mucin. Within the glands, the cell population usually showed normal differentiation. A short zone of replicating cells was found at the base of the crypt. Most of the gland was lined by goblet cells. Thin bundles of smooth muscle were occasionally identified between the glandular structures.

Dr. Rukunuzzaman: A patient who has only a few uncomplicated polyps should be managed with endoscopic polypectomy by single or multiple settings. If a juvenile polyp shows high-grade dysplasia and the polyp cannot be completely removed endoscopically, or if invasive adenocarcinoma is detected, then consideration should be given to colectomy. In those patients with large numbers of juvenile polyps, especially those with significant anemia or hypoproteinemia, subtotal colectomy with ileorectal anastomosis is recom- mended, or proctocolectomy with J pouch ileo-anal anastomosis.12 A few gastric polyps may be managed with endoscopic polypectomy, but in case of multiple or diffuse gastric polyposis, subtotal or total gastrectomy may be necessary. Duodenal or small bowel polyposis may need removal surgically. 3 Lower gastrointestinal endoscopic surveyllance should be done annually until polyp free, then 3 yearly. Family members at risk should be screened from 15 years of age by upper and lower gastrointestinal endoscopy. If normal then repeat endoscopy 3 yearly. If polyp found remove and screen yearly. $\underline{12}$ The presenting case was managed by polypectomy endoscopically by multiple setting and other nutritional support. Though initial histopathology report of polyps showed hamartomatous polyp without dysplasia. But on the subsequent setting of colonoscopy, polyps sent for histopathology which shows tubular adenoma with mild dysplasia (Figure 2). Agnifili et al. (1999) showed the overall incidence of adenomas were found in 50 patients $(18.5 \%)$, carcinomas were found in 43 patients $(17.3 \%)$ among 271 juvenile polyposis patients. In the 50 patients with adenomatous changes, 48 were colorectal adenomas. 21 Genetic counseling was done and also advice for endoscopic surveillance of other offsprings from 15 years of age.

Dr. Rubaiyat Alam: Whether it may be a case of Turner syndrome?

Dr. A. S. M. Bazlul Karim: Turner's syndrome is genetic syndrome results from loss of all or part of sex chromosome. It is characterized by female phenotype, short stature, webbed neck, learning difficulties, pubertal delay and gonadal dysgenesis. Other associated congenital malformations with the XO chromosomal karyotype are horseshoe kidney, coarctation of the aorta and a variety of skeletal abnormalities.22 Turner's syndrome patient may present with gastrointestinal bleeding if associated with inflammatory bowel disease, intestinal telangiectasia or hemangioma. $\underline{23}$ These patients also present with gastrointestinal bleeding, short stature, absent secondary sexual characters and infantile uterus which suggest the diagnosis. But the presence of gastrointestinal polyps and normal karyotyping exclude the diagnosis.

Dr. Kamal Hossen: Why it is not the Peutz-Jegher syndrome?

Dr. Yasmin: Peutz-Jeghers syndrome is one of the autosomal dominant hamartomatous intestinal poly -posis syndromes. The extraintestinal features are mucocutaneous skin pigmentation of variable size with a cluster around the mouth, nostrils, eyes and the peri-anal areas. It may also be found on the fingers and toes, the dorsal and ventral aspects of the hands and feet rarely. $\underline{24}$ Polyps are preferentially located in small intestine (commonly jejunum than 
ileum and duodenum or may affect stomach and colorectum). $\underline{25}$ Diagnostic criteria for Peutz-Jeghers syndrome by Giardello is histopathological evidence of hamartomatous gastrointestinal polyps and two of the following criteria: positive family history, pigmented skin or mucosal brown macules and small bowel polyposis.26 Microscopically, there is arborizing network of connective tissues and smooth muscle extending through the core of the polyp surrounded by intestinal glands lined by normal intestinal epithelium. 27 Masson's trichrome stain demonstrated the presence of diagnostic smooth muscle within the central core of the polyp. Histologically, it is hamatomatous polyp like other hamartomatous polyposis syndromes with hyperplasia of smooth muscle layer which extends in tree like arborising manner toward the epithelial layer which is absent in others. In the presenting case, there was no mucocutaneous pigmentation. Distribution of polyps and histopathology features were also against Peutz-Jegher syndrome.

Dr. Shashi Bhushan: Is there any need for small bowel evaluation for polyps?

Dr. Rukunuzzaman: The commonly affected sites of polyps in juvenile polyposis patients, in order of frequency are colorectum (98\%), stomach $(14 \%)$, jejunum plus ileum $(7 \%)$ and duodenum (2\%). 15 Though small bowel is less commonly affected, it can be seen by enteroscopy or CT enterography. The procedures are not available in Bangladesh.

Dr. Ruhina Tasneem: As it is an autosomal dominant genetic syndrome, is the mutation analysis necessary?

Dr. Karim: Juvenile polyposis syndrome families have identified two specific genemutations: SMAD4 and BMPR1A. Upper gastrointestinal polyps are more frequent in patients with SMAD4+ mutation compared with mutation-negative cases, and family members with upper gastrointestinal polyps have higher prevalence compared with BMPR1A and mutation-negative cases. The frequency of a family history of gastrointestinal cancer was significantly higher for mutation positive group (89\%) compared to mutation negative group (52\%). .3 So, mutation analysis necessary for confirmation, to predict the prognosis and also for the screening of risk groups. We could not do due to unavailability of the test in our country.

Dr. Zannatul Ferdous Sonia: What is the prognosis of this patient?

Dr. Karim: It is a premalignant condition. The incidence of colorectal cancer is $20.7 \%$ by the age 34 years. 5 The cumulative lifetime risk of colorectal cancer is as high as $68 \%$ at the age of 60 year. $\underline{28}$ Most striking features of the gastric polyp are anemia $(89 \%)$ and hypoproteinemia $(67 \%)$ requiring gastrictomy. This patient may develop colorectal carci- noma and need colectomy as last histopathology report showed tubular adenoma with mild dysplasia. The incidence of gastric adenocarcinoma is $21 \%$ in juvenile polyposis syndrome patients who have gastric polyp. $\underline{29}$ Other associated anomalies are cardiovascular system and genitourinary abnormalities resulting in morbidity and mortality. Our patient has both colorectal and gastric polyp. Regular follow-up may increase the life expectancy by recognizing early complications. She is also on follow-up without medication for ovarian failure.

\section{Final Diagnosis}

Juvenile polyposis syndrome

\section{References}

1. Fakhoury M, Negrulj R, Mooranian A, Al-Salami H. Inflammatory bowel disease: Clinical aspects and treatments. J Inflamm Res. 2014; 7: 113-20.

2. Islam S, Agarwal M, Talukdar AJ, Dutta S, Kalita PP. A study on clinical profileof patients with inflammatory bowel disease. NJMR. 2016; 6: 233-36.

3. Chow E, Macrae F. Review of juvenile polyposis syndrome. J Gastroenterol Hepatol. 2005; 20: 163440.

4. Brosens LA, van Hattem WA, Jansen M, de Leng WW, Giardiello FM, Offerhaus GJ. Gastrointestinal polyposis syndromes. Curr Mol Med. 2007; 7: 29 46.

5. Jass JR, Williams CB, Bussey HJ, Morson BC. Juvenile polyposis: A precancerous condition. Histopathology 1988; 13: 619-30.

6. Zbuk KM, Eng C. Hamartomatous polyposis syndromes. Nat Clin Pract Gastroenterol Hepatol. 2007; 4: 492-502.

7. Eng C. Will the real Cowden syndrome please stand up: Revised diagnostic criteria? J Med Genet. 2000; 37: 828-30.

8. Gorlin RJ, Cohen MM Jr, Condon LM, Burke BA. Bannayan-Riley-Ruvalcaba syndrome. Am J Med Genet. 1992; 44: 307-14.

9. Jones K (ed). Smith's recognizable patterns of human malformation. Vol. 5. Philadelphia, WB Saunders, 1997.

10. McColl I, Busxey HJ, Veale AM, Morson BC. Juvenile polyposis coli. Proc R Soc Med. 1964; 57: 89697.

11. Schreibman IR, Baker M, Amos C, McGarryty TJ. The hamartomatous polyposissyndromes: A clinical and molecular review. Am J Gastroenterol. 2005; 100: 476-90.

12. Howe JR, Sayed MG, Ahmed AF, Ringold J, Larsen -Haidle J, Merg A, Mitros FA, Vaccaro CA, Petersen 
GM, Giardiello FM, Tinley ST. The prevalence of MADH4 and BMPR1A mutations in juvenile polyposis and absence of BMPR2, BMPR1B, and ACVR1 mutations. J Med Genet. 2004; 41: 484-91.

13. Cichy W, Klincewicz B, Plawski A. Juvenile polyposis syndrome. Arch Med Sci. 2014; 10: 570-77.

14. Alam S, Islam KMD, Mohammad N, Nooruzzaman M. Per-rectal bleeding in children: Experiences in the Department of Paediatric Surgery in BSMMU. Bangladesh Med Res Counc Bull. 2017; 43: 20-25.

15. Poddar U, Thapa BR, Vaiphei K, Singh K. Colonic polyps experience of 236 Indian children. Am J Gastroenterol. 1998; 93: 619-22.

16. Sachatello CR, Hahn IS, Carrington CB. Juvenile gastrointestinal polyposis in a female infant: Report of a case and review of the literature of a recently recognized syndrome. Surgery 1974; 75: 107-14.

17. Hofting I, Pott G, Stolte M. The syndrome of juvenile polyposis. Leber Magen Darm. 1993; 23: 111-12.

18. Rozen P, Samuel Z, Brazowski E, Jakubowicz M, Rattan J, Halpern Z. An audit of familial juvenile polyposis at the Tel Aviv Medical Center: Demographic, genetic and clinical features. Familial Cancer. 2003; 2: 1-7.

19. Coburn MCPV, DeLuca FG, Bland KI. Malignant potential in intestinal juvenile polyposis syndromes. Ann Surg Oncol. 1995; 2: 386-91.

20. Perzin KH, Bridge MF. Adenomatous and carcinomatous changes in hamartomatous polyps of the small intestine (Peutz-Jeghers syndrome): Report of a case and review of the literature. Cancer 1982; 49: 971-83.

21. Agnifili A, Verzaro R, Gola P, Marino M, Mancini E, Carducci G, Ibi I, Valenti M. Juvenile polyposis: Case report and assessment of the neoplastic risk in 271 patients reported in the literature. Dig Surg.
1999; 16: 161-66.

22. Lisser H, Curtis LE, Escamilla RF, Goldberg MB The syndrome of congenitally aplastic ovaries with sexual infantilism, high urinary gonadotropins, short stature, and other congenital abnormalities. J Clin Endocrinol. 1947; 7:665-70.

23. Eroglu Y, Emerick KM, Chou PM, Reynolds M. Gastrointestinal bleeding in Turner's Syndrome: A case report and literature review. J Pediatr Gastroenterol Nutr. 2002; 35: 84-87.

24. Lindor NM, Greene MH. Mayo Familial Cancer Program: The concise handbook of family cancer syndromes. J Natl Cancer Inst. 1998; 90: 1039-41.

25. Utsunomiya J, Gocho H, Miyanaga T, Hamaguchi E. Peutz-Jeghers syndrome: Its natural course and management. Johns Hopkins Med J. 1975; 136: 7182.

26. Giardiello FM, Welsh SB, Hamilton SR, Offerhaus GJ, Gittelsohn AM, Booker SV, Krush AJ, Yardley $\mathrm{JH}$, Luk GD. Increased risk of cancer in the PeutzJeghers syndrome. N Engl J Med. 1987; 316: 151114.

27. Choudhury T, Enam S, Begum F, Taufiq F, Kamal M. Peutz-Jeghers polyp: A retrospective study on twelve cases received at the Department of Pathology, Bangabandhu Sheikh Mujib Medical University. Bangabandhu Sheikh Mujib Med Univ J. 2012; 5: 12-17.

28. Desai DC, Neale KF, Talbot I.C, Hodgson SV, Phillips RK. Juvenile polyposis. Br J Surg. 1995; 82: 14-17.

29. Giardiello FM, Hamilton SR, Kern SE, Offerhaus GJ, Green PA, Celano P, Krush AJ, Booker SV. Colorectal neoplasia in juvenile polyposis or juvenile polyps. Arch Dis Child. 1991; 66: 971-75. 